\title{
Synthesis and Optimization of Almost Periodic Antennas Using Floquet Modal Analysis and MoM-GEC Method
}

\author{
Nader Ben Latifa, Taoufik Aguili \\ Laboratory of the Communication Systems, National Engineering School of Tunis, Le Blvedere, Tunis, Tunisia \\ Email: benlatifanader@yahoo.fr, taoufik.aguili@gmail.com
}

How to cite this paper: Latifa, N.B. and Aguili, T. (2019) Synthesis and Optimization of Almost Periodic Antennas Using Floquet Modal Analysis and MoM-GEC Method. Journal of Electromagnetic Analysis and Applications, 11, 1-16.

https://doi.org/10.4236/jemaa.2019.111001

Received: December 29, 2018

Accepted: January 27, 2019

Published: January 30, 2019

Copyright (๑) 2019 by author(s) and Scientific Research Publishing Inc. This work is licensed under the Creative Commons Attribution International License (CC BY 4.0).

http://creativecommons.org/licenses/by/4.0/

\begin{abstract}
This paper presented a new Floquet analysis used to calculate the radiation for 1-D and 2-D coupled periodic antenna systems. In this way, an accurate evaluation of mutual coupling can be proven by using a new mutual interaction expression that was based on Fourier analysis. Then, this work indicated how Floquet analysis can be used to study a finite array with uniform amplitude and linear phase distribution in both $x$ and $y$ directions. To modelize the proposed structures, two formulations were given in a spectral and spatial domain, where the Moment (MoM) method combined with a generalized equivalent circuit (GEC) method was applied. Radiation pattern of coupled periodic antenna was shown by varying many parameters, such as frequencies, distance and Floquet states. The 3-D radiation beam of the coupled antenna array was analyzed and compared in several steering angles $\theta_{s}$ and coupling values $d_{x}$. The simulation of this structure demonstrated that directivity decreased at higher coupling values. The secondary lobs in the antenna radiation pattern affected the main lobe gain by energy dispersal and considerable increasing of side lobe level (SLL) may be achieved. Therefore, the sweeping of the radiation beam in several steering directions affected the electromagnetic performance of the antenna system: the directivity at the steering angle $\theta_{s}=\pi / 3$ was more damaged and had $19.99 \mathrm{~dB}$ while the second at $\theta_{s}=0$ had about $35.11 \mathrm{~dB}$. This parametric study of coupled structure used to concept smart periodic antenna with sweeping radiation beam.
\end{abstract}

\section{Keywords}

Periodic Antennas, Floquet Theory, Moment Method, GEC Method

\section{Introduction}

From the early days of communication systems, antenna arrays have been wide- 
spread. They are used in base stations, mobile phones and radars. They are made by elementary antennas combined together to synthesize a radiation pattern with a directional beam. There are several conventional methods applied to analyze these systems in [1] [2] [3]. The coupling between the radiating elements increases with the dimension of the antenna arrays, making it one of the most important factors in the choice of the analysis method. Similar examples are found in [4]. In this context, the robustness of such method is evaluated by its resistance in front of the interaction between the radiating elements. In this work, we present a new formulation of the MoM-CEG method based on the Floquet theorem, (refer to [5] [6] [7] for details). This Floquet approach shows that the electromagnetic field distribution in periodic structures changes only by multiplication of a complex constant for a translation by one period in the global structure. The electromagnetic compilation of antenna arrays with an $\mathrm{N}$ pattern is then reduced to a calculation on one reference cell with periodic walls in a new modal base. Indeed, the term of phase shift contains itself the electromagnetic information of other adjacent cells. These artificial walls are implemented to group all phases from the periodic structure [8] [9] [10] [11]. Then, the Floquet modal analysis brings back all possible space calculations to new model calculation. Then, the Floquet modal analysis examines all the Floquet states and the coupling information of the overall structure. This new method is applied to generate a 3-D electronically controlled antenna. The sweeping of the radiation beam of smart antennas is nowadays used to improve the performance of mobile and wireless communications systems [12] [13]. A number of works have been reported taking different periodic structures with spectral formulation to study 1-D and 2-D periodic antenna array [14] [15] [16] [17]. But this work presents a new formulation of the MoM-CEG method based on Floquet theorem to optimize coupled periodic antenna with sweeping radiation beam.

This paper is organized as follows: First it is necessary to explain how to use Floquet modal analysis to decrease the complexity of periodic 1-D and 2-D structures, see e.g. [14]-[19]. Next, we present the numerical results and discuss the behavior of a 3-D steerable radiation beam and how to concept smart periodic antenna with sweeping beam with optimal performance.

\section{Problem Formulation: Periodic Antenna Array}

\subsection{Formulation of 1D Periodic Antenna Array}

In this section the formulation of the problem is illustrated in detail. A Floquet theory is proposed to reduce the infinite domain to a single cell with periodic walls. An electrical field is then formulated and solved through a MoM-GEC [20] [21] approach in a spectral domain [22] [23] [24] [25]. The structure under analysis is shown in Figure 1. The excitation is given by an $E_{0}$ voltage source placed in the middle of a metallic patch. The width and the length of patches are $w$ and $l$. The spatial period along the $x$ direction is $d_{x}$. The height of dielectric substance is $h$, and its relative permittivity $\varepsilon_{r}$ is mounted on aground plane.

This structure is taken as infinite in $( \pm X)$ and periodic with a period $d_{x}$. 


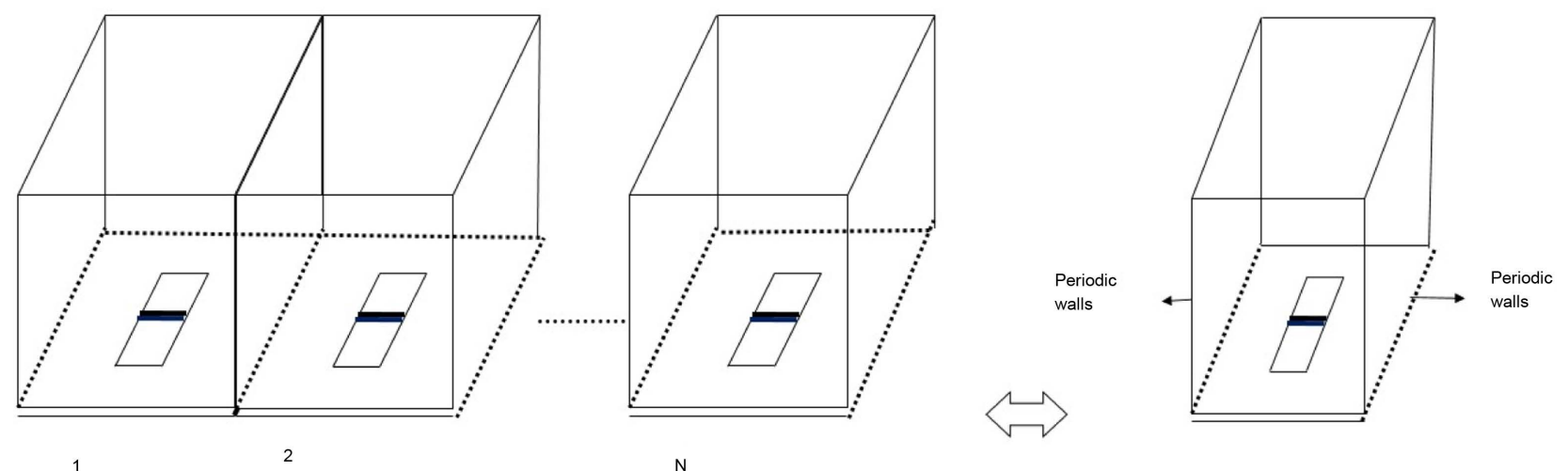

Figure 1. 1-D periodic antenna arrays presentation with Floquet theory.

Floquet theorem can be used with this geometric periodicity, so the study of global structure is reduced to one cell with Floquet phases $\exp \left(j \alpha N d_{x}\right)$. For details, see [2] [3] [10] [26] [27]. $\tilde{E}(x)$ represents an electric field reacting with this periodicity.

$$
\left\{\begin{array}{l}
\tilde{E}\left(x+d_{x}\right)=\exp \left(j \alpha d_{x}\right) * \tilde{E}(x) \\
\tilde{E}\left(x+2 d_{x}\right)=\exp \left(j \alpha 2 d_{x}\right) * \tilde{E}(x) \\
\tilde{E}\left(x+N d_{x}\right)=\exp \left(j \alpha N d_{x}\right) * \tilde{E}(x)
\end{array}\right.
$$

Each Floquet phase corresponds to a Floquet state, and the function $F_{\alpha m}$ characterizes all possible states.

$$
F_{\alpha m}=\frac{1}{d_{x}} \exp (j \alpha x) * \exp \left(j \frac{2 \Pi m x}{d_{x}}\right)
$$

where $\alpha$ and $\mathrm{m}$ correspond respectively to Floquet mode and spectral domain mode. The $\alpha$ values are in Brillouin domain $\left[\frac{-\Pi}{d_{x}}, \frac{\Pi}{d_{x}}\right]$. And for $\mathrm{N}$ discrete values of $\alpha, \alpha_{p}$ are given by:

$$
\alpha_{p}=\frac{2 \Pi p}{L}
$$

where $\frac{-N}{2} \leq p \leq \frac{N}{2}-1$ and $L=N * d_{x}$.

The electric field of the central cell in spacial domain is $\tilde{E}_{m}$. We associate the electric field $\tilde{E}_{\alpha}$ in spectral domain, which models all waves emitted from other cells of periodic structure.

$$
\tilde{E}_{m+1}=\tilde{E}_{m} \exp \left(j \alpha d_{x}\right)
$$

Then

$$
\tilde{E}_{m}=\frac{d_{x}}{2 \Pi} \int_{\frac{\Pi}{d_{x}}}^{\frac{\Pi}{d_{x}}} \tilde{E}_{\alpha} \exp \left(j \alpha m d_{x}\right) \mathrm{d} \alpha
$$

The spectral domain MoM-GEC technique can be applied for this single cell with periodic walls to extract the electromagnetic parameter. The pertinent 
problem of the use an electric field integral equation can be solved by applying the GEC method. It can replace the integral equation by a simple equivalent circuit in the discontinuity surface and applies the laws of tension and current to extract the relation between electric and current field by using an admittance operator [2] [3] [14] [15]. The discontinuity surface contains metallic and dielectric parties. The equivalent circuit of the unit cell is shown in Figure 2. The virtual electric field is defined on the metallic surface and is null on the dielectric part. We note that $E_{e \alpha}$ its dual. Similar examples are found in [28] [29] [30].

From this circuit, we can deduce this system:

$$
\left\{\begin{array}{l}
\tilde{J}_{e \alpha}=\tilde{J}_{\alpha} \\
\tilde{E}_{e \alpha}=-\tilde{E}_{0 \alpha}+\frac{1}{\hat{Y}_{\alpha}^{e q}} * \tilde{J}_{e \alpha}
\end{array}\right.
$$

The equivalent admittance operator is:

$$
\hat{Y}_{\alpha}^{e q}=\hat{Y}_{\alpha}^{\text {upper }}+\hat{Y}_{\alpha}^{\text {down }}
$$

$\hat{Y}_{\alpha}^{\text {upper }}$ is the upper admittance operator of the infinite empty wave guide with periodic walls, and $\hat{Y}_{\alpha}^{\text {down }}$ is the down admittance operator of the short circuited dielectric wave guide of height $h$ with periodic walls.

$$
\begin{aligned}
\hat{Y}_{\alpha}^{\text {upper }} & =\sum_{m n}\left|f_{m n}\right\rangle y_{m n \alpha}^{\text {upper }}\left\langle f_{m n}\right| \\
\hat{Y}_{\alpha}^{\text {down }} & =\sum_{m n}\left|f_{m n}\right\rangle y_{m n \alpha}^{\text {down }}\left\langle f_{m n}\right|
\end{aligned}
$$

$f_{m n}$ are the base propagation mode functions.

Next, we apply the Galerkin method, where we project the excitation mode $f_{m n}$ and the test function $g_{p q}$ on the previous equation. We then have the following system:

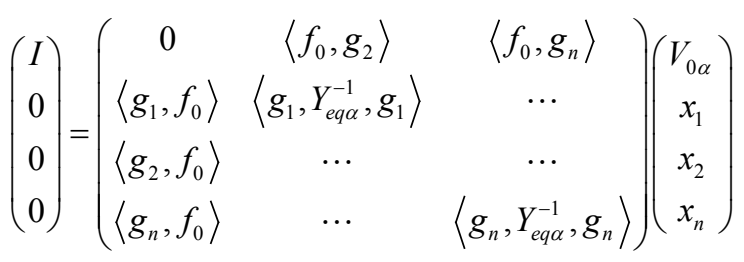

The matrix form of the former equation can be developed as following:

$$
\left(\begin{array}{c}
I_{0 \alpha} \\
0
\end{array}\right)=\left(\begin{array}{cc}
0 & A^{t} \\
-A^{t} & B
\end{array}\right)\left(\begin{array}{c}
V_{0 \alpha} \\
X
\end{array}\right)
$$

where $A$ is the excitation vector and $B$ is the coupling matrix. The test courant functions in metallic part are $g_{p q}$.

The resolution of the previous system consequently helps to calculate the virtual electric field $E_{e \alpha}$ and the electric far field $\tilde{E}_{R a d}$ of the coupled structure.

\subsection{Formulation 2-D Periodic Antenna Arrays}

We take the example of 2-D planar periodic structures of $d_{x}$ periodicity along 


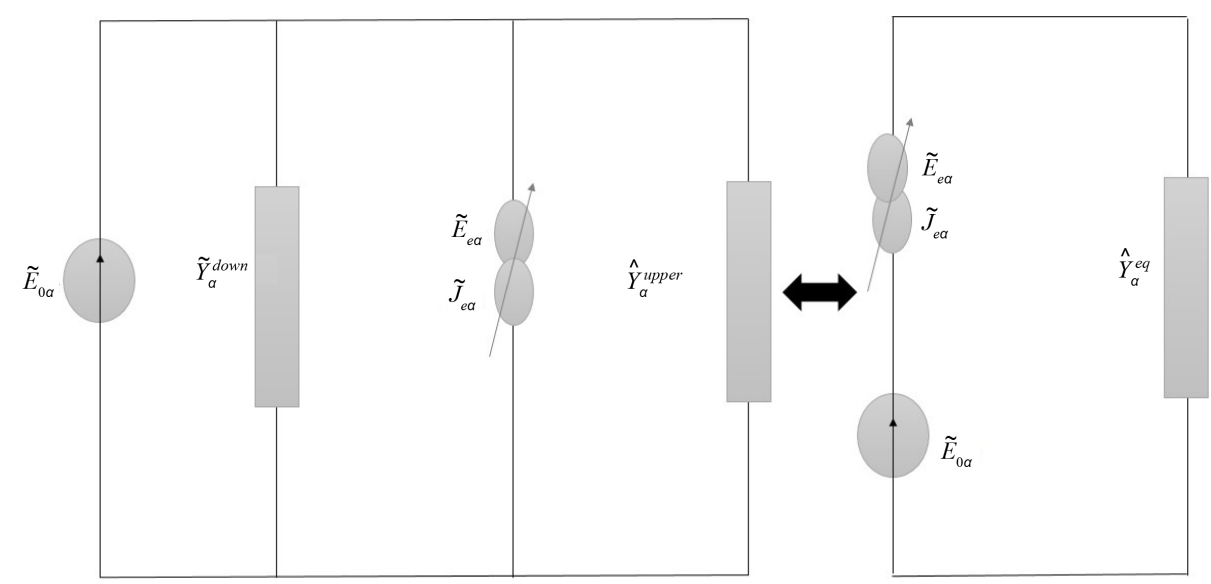

Figure 2. Equivalent circuit in spectral domain with MoM-CEM method of unit cell.

the $x$-axis and $d_{y}$ periodicity along the $y$-axis in Figure 3. Floquet theorem can be applied to synthesize periodic structures where all antenna elements are coupled with each other [16] [17] [31].

The formulation of the MoM-CEM method is applied to this reference cell with periodic walls in modal space, and the distribution of magnetic field differs only by two phases $(\alpha, \beta)$ compared to other cells. We pose $J_{i, j}$ the source field in $(i, j)$ base cell in periodic device. In this new modal base, $J_{\alpha, \beta}$ is the set of excitation of the others cells such as:

$$
\left\{\begin{array}{l}
J_{i+1, j+1}=J_{i, j} \exp \left(i \alpha d_{x}\right) \exp \left(i \beta d_{y}\right) \\
J_{i+1, j}=J_{i, j} \exp \left(i \alpha d_{x}\right) \forall i, j \\
J_{i, j+1}=J_{i, j} \exp \left(i \beta d_{y}\right)
\end{array}\right.
$$

Floquet modal analysis reduces spacial electromagnetic calculus of 2-D periodic structure to a spectral calculus in a new modal base which gathers all possible phases in periodic walls. In this case, we consider the 2-D dimensional case along the $x$ - and $y$-axis with $(N * N)$ identical cells, where each one is excited by a located source. The two phases $\alpha$ and $\beta$ belong respectively to the Brillouin domain: $\left[\frac{-\Pi}{d_{x}}, \frac{\Pi}{d_{x}}\right]$ and $\left[\frac{-\Pi}{d_{y}}, \frac{\Pi}{d_{y}}\right]$. The discretization of Floquet mode provides the following: $\alpha=2 \pi p / L$ and $\beta=2 \pi q / L$, where $p$ and $q$ are two integer and $L=N * d$. From these Floquet phases, we associate two fields $E_{\alpha \beta}$ and $J_{\alpha \beta}$ which model all waves emitted from others cells of the periodic structure.

The discontinuity surface contains metallic and dielectric parties. The excitation $E_{\alpha \beta}$ of the central path produces a current field $J_{\alpha \beta}$. This virtual magnetic field $J_{e \alpha \beta}$ is defined on the metallic surface and is null on the dielectric part. We note that $E_{e \alpha \beta}$ is its dual. The electric field $J_{m n}$ can be developed as following:

$$
J_{m n}=d / 2 \pi \int_{-d / \pi}^{d / \pi} \int_{-d / \pi}^{d / \pi} \tilde{J}_{\alpha \beta} \exp (-j \alpha m d) \exp (-j \beta n d) \mathrm{d} \alpha \mathrm{d} \beta
$$




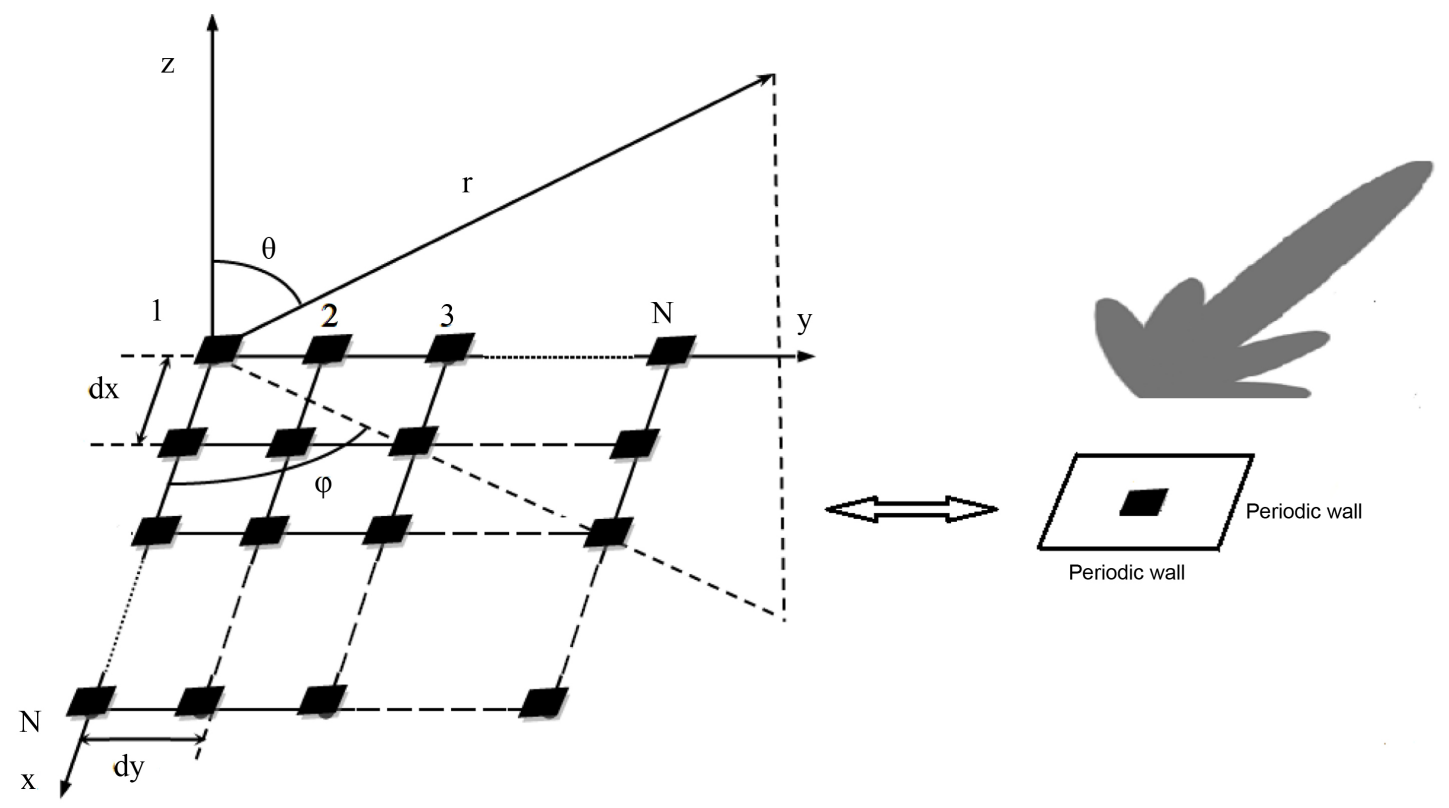

Figure 3. 2-D periodic antenna arrays presentation with Floquet theory.

which $d / 2 \pi$ is a normalization factor and $d_{x}=d_{y}=d$. Based on the MoM-CEM method and using the laws of tension and current of the equivalent circuit, we can extract and identify the relationship between the current density, the electric field and admittance operator $\hat{Y}_{\alpha \beta}^{e q}$.

From this equivalent circuit, we can deduce the following system:

$$
\left\{\begin{array}{l}
\tilde{J}_{\alpha \beta}=\tilde{J}_{e \alpha \beta} \\
\tilde{E}_{e \alpha \beta}=-\tilde{E}_{0 \alpha \beta}+\frac{1}{\hat{Y}_{\alpha \beta}^{e q}} * \tilde{J}_{e \alpha \beta}
\end{array}\right.
$$

Then

$$
\left(\begin{array}{c}
J_{\alpha \beta} \\
E_{e \alpha \beta}
\end{array}\right)=\left(\begin{array}{cc}
0 & 1 \\
-1 & \frac{1}{\hat{Y}_{\alpha \beta}^{e q}}
\end{array}\right)\left(\begin{array}{c}
E_{0 \alpha \beta} \\
J_{e \alpha \beta}
\end{array}\right)
$$

The equivalent admittance operator is:

$$
\hat{Y}_{\alpha \beta}^{e q}=\hat{Y}_{\alpha \beta}^{\text {down }}+\hat{Y}_{\alpha \beta}^{u p p e r}
$$

$\hat{Y}_{\alpha \beta}^{\text {upper }}$ and $\hat{Y}_{\alpha \beta}^{\text {down }}$ are respectively the upper and the down admittance operator.

where:

$$
\begin{aligned}
& \hat{Y}_{\alpha \beta}^{\text {upper }}=\sum_{m n}\left|f_{m n}\right\rangle y_{m n \alpha \beta}^{\text {upper }}\left\langle f_{m n}\right| \\
& \hat{Y}_{\alpha \beta}^{\text {down }}=\sum_{m n}\left|f_{m n}\right\rangle y_{m n \alpha \beta}^{\text {down }}\left\langle f_{m n}\right|
\end{aligned}
$$

$f_{m n}$ are the base propagation mode functions.

The previous matrix presentation is projected under base and test functions 
$\left(f_{m n, g_{p q}}\right)$ and can be developed as the following:

$$
\left(\begin{array}{l}
I \\
0 \\
0 \\
0
\end{array}\right)=\left(\begin{array}{ccc}
0 & \left\langle f_{0}, g_{2}\right\rangle & \left\langle f_{0}, g_{n}\right\rangle \\
\left\langle g_{1}, f_{0}\right\rangle & \left\langle g_{1}, Y_{\text {eq } \alpha \beta}^{-1}, g_{1}\right\rangle & \ldots \\
\left\langle g_{2}, f_{0}\right\rangle & \ldots & \ldots \\
\left\langle g_{n}, f_{0}\right\rangle & \ldots & \left\langle g_{n}, Y_{\text {eq } \alpha \beta}^{-1}, g_{n}\right\rangle
\end{array}\right)\left(\begin{array}{c}
V_{0 \alpha \beta} \\
x_{1} \\
x_{2} \\
x_{n}
\end{array}\right)
$$

So, we can deduce the following system:

$$
\left(\begin{array}{c}
I_{0 \alpha \beta} \\
0
\end{array}\right)=\left(\begin{array}{cc}
0 & A^{t} \\
-A^{t} & B
\end{array}\right)\left(\begin{array}{c}
V_{0 \alpha \beta} \\
X
\end{array}\right)
$$

where $A$ is the excitation vector and $B$ is the coupling matrix.

\section{Results and Observations}

\subsection{Studie of Floquet States}

In this section, we present a Floquet modal analysis of periodic antenna array. As an example we simulate and design a structure of four linear elements using matlab software. We extract all possible Floquet modes $(\alpha, \beta)$; and we show their influence on pattern radiation. Results are presented for the following parameters: $\varepsilon_{r}=1, h=5.5 \mathrm{~mm}, d_{x}=108 \mathrm{~mm}, d_{y}=54 \mathrm{~mm}, l=27 \mathrm{~mm}, w=1$ and $\delta=0.75 \mathrm{~mm}$.

The behavior of magnetic field for one reference cell is shown in Figure 4. Table 1 illustrates the performance parameter (side lobe level, peak gain and directivity) of radiation pattern for each Floquet mode. Refer to [32] [33] [34] for details. The radiation pattern plot in Figure 5 obtained by using Floquet modal method demonstrates the aptitude of this technique to superpose all Floquet modes $\left(\alpha_{-2}, \alpha_{-1}, \alpha_{0}, \alpha_{1}\right)$ and their superposition $E_{t o t}$ for different steering angles $\theta_{s}$ [35] [36].

The electromagnetic parameters of each Floquet mode $\left(\alpha_{-2}, \alpha_{-1}, \alpha_{0}, \alpha_{1}\right)$ of this periodic structure are compared in Table 1 . The simulation of this periodic structure demonstrates that each Floquet mode defines the mutual coupling of the adjacent cell. The superposition of all Floquet modes represents the evolution of pattern radiation of the reference cell coupled with all others cells.

\subsection{Frequency and Coupling Effect}

In this section, we present and briefly discuss several results of the radiation pattern on an open structure analyzed in the previous section. The simulated radiation

Table 1. Performance Parameters of the 1-D periodic structure for each Floquet mode.

\begin{tabular}{cccc}
\hline Floquet mode & SLL $(\mathrm{dB})$ & Peak gain $(\mathrm{dBi})$ & Directivity $(\mathrm{dB})$ \\
\hline$\alpha_{1}$ & -13 & -6.1 & 25.18 \\
$\alpha_{0}$ & -23 & -11.4 & 23.66 \\
$\alpha_{-1}$ & -13 & -6.1 & 25.81 \\
$\alpha_{-2}$ & -11.5 & -9.6 & 25.42 \\
\hline
\end{tabular}




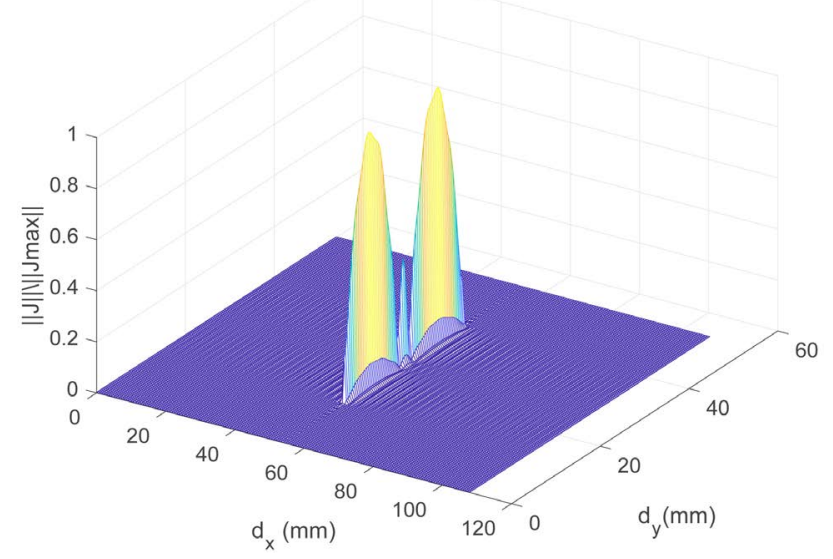

Figure 4. 2-D representation of the current density $\|J\| /\|J \max \|$ with the basis functions at $F=5.4 \mathrm{Ghz}, h=1.25 \mathrm{~mm}, d_{x}=108 \mathrm{~mm}, d_{y}=54 \mathrm{~mm}, w=1 \mathrm{~mm}, \epsilon=1, \epsilon_{r}=1$, $\delta=0.75 \mathrm{~mm}, \lambda=54 \mathrm{~mm}, \quad \alpha=0 \mathrm{rad} \cdot \mathrm{m}^{-1}, \quad \beta=0 \mathrm{rad} \cdot \mathrm{m}^{-1}$.

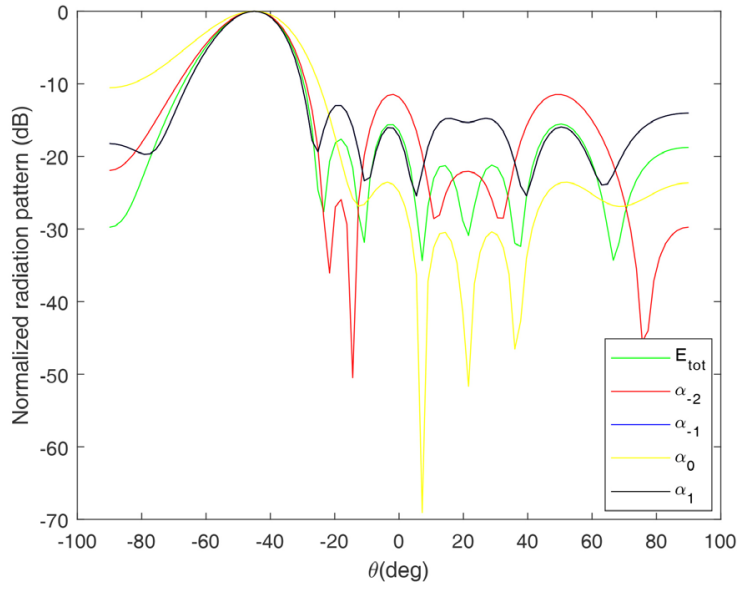

(a)

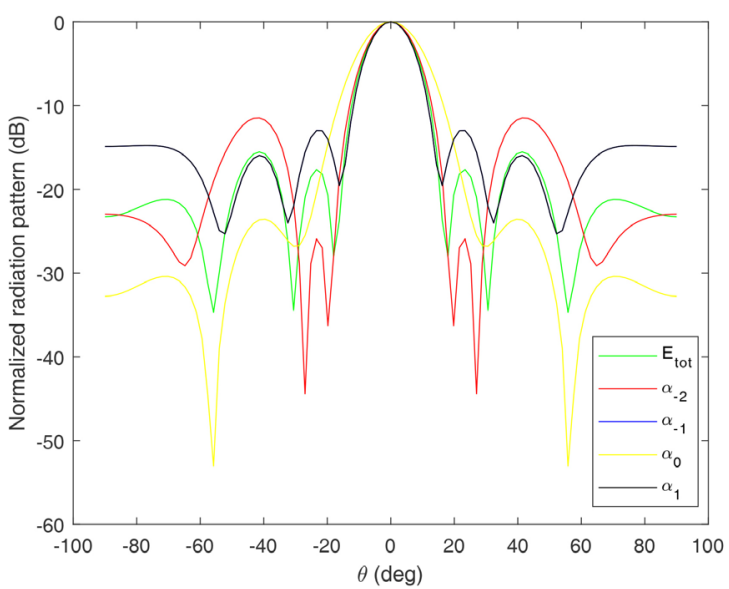

(b)

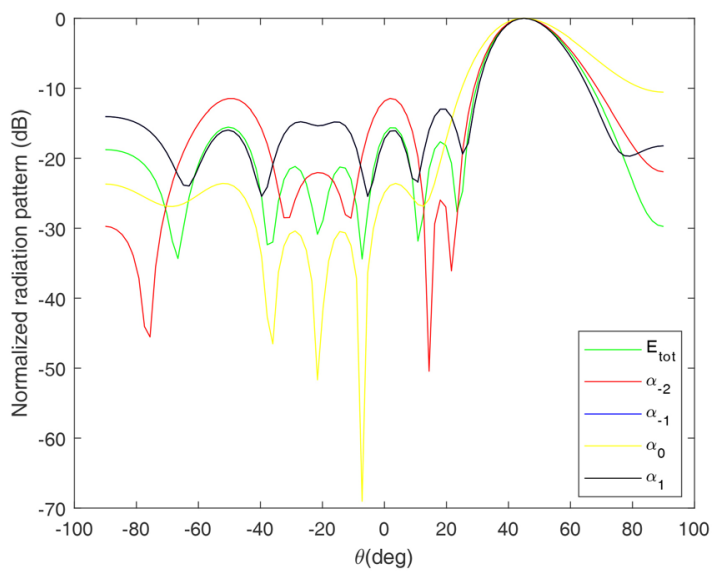

(c)

Figure 5. Simulated 2-D normalized radiation pattern of periodic antenna for several steering angles $\theta_{s}$ using: $F=5.4 \mathrm{Ghz}, h=1.25 \mathrm{~mm}, d_{x}=108 \mathrm{~mm}, d_{y}=54 \mathrm{~mm}, w=1 \mathrm{~mm}, \epsilon=1, \epsilon_{r}=1, \delta=0.75 \mathrm{~mm}, \lambda=54 \mathrm{~mm}$. (a) $\theta_{s}=-45 \mathrm{deg}$, (b) $\theta_{s}=0 \mathrm{deg},(\mathrm{c}) \theta_{s}=45 \mathrm{deg}$. 
pattern of the antenna is shown in Figure 6. The electromagnetic near fields of the planner antenna were simulated with Floquet analysis combined with MoM-CEG method. From these results, the radiation pattern was calculated using the conventional method of the stationary phase. It can be observed that the evolution of the pattern radiation for different values of the frequency from 4 to $10 \mathrm{GHz}$ is not the same, but they have a similar behavior. By exploiting this figure, at $F=4 \mathrm{GHz}$, a low directivity is achieved with high power Pmax (the power in decibel between the maximum of main lobe and the maximum of secondary lobs) with $40 \mathrm{~dB}$ and at $10 \mathrm{GHz}$, a high directivity is achieved with low power Pmax with $10 \mathrm{~dB}$. We deduce then that the diagram becomes increasingly selective by increasing the frequency, but at the same time, the amplitude of the secondary lobes increases.

Figure 7 shows the influence of coupling value on radiation pattern in relation to wavelength. For $d_{x}=\lambda$, the power Pmax is $30 \mathrm{~dB}$, but for $d_{x}=3 \lambda$, the power Pmax is $10 \mathrm{~dB}$. From these results, we can deduce that the pattern radiation of this structure becomes more directive when the period $\mathrm{dx}$ increases compared to wavelength $\lambda$.

In this example, on 1-D periodic array four planar antennas is discussed. The amplitude of each excitation and the phase difference of neighboring cell are identicals. The radiation simulation shown in Figure 6 and Figure 7 reveals that the behavior of radiation pattern is acceptable with MoM-GEC method combined to Floquet theory and large separation between elements is needed for high directivity.

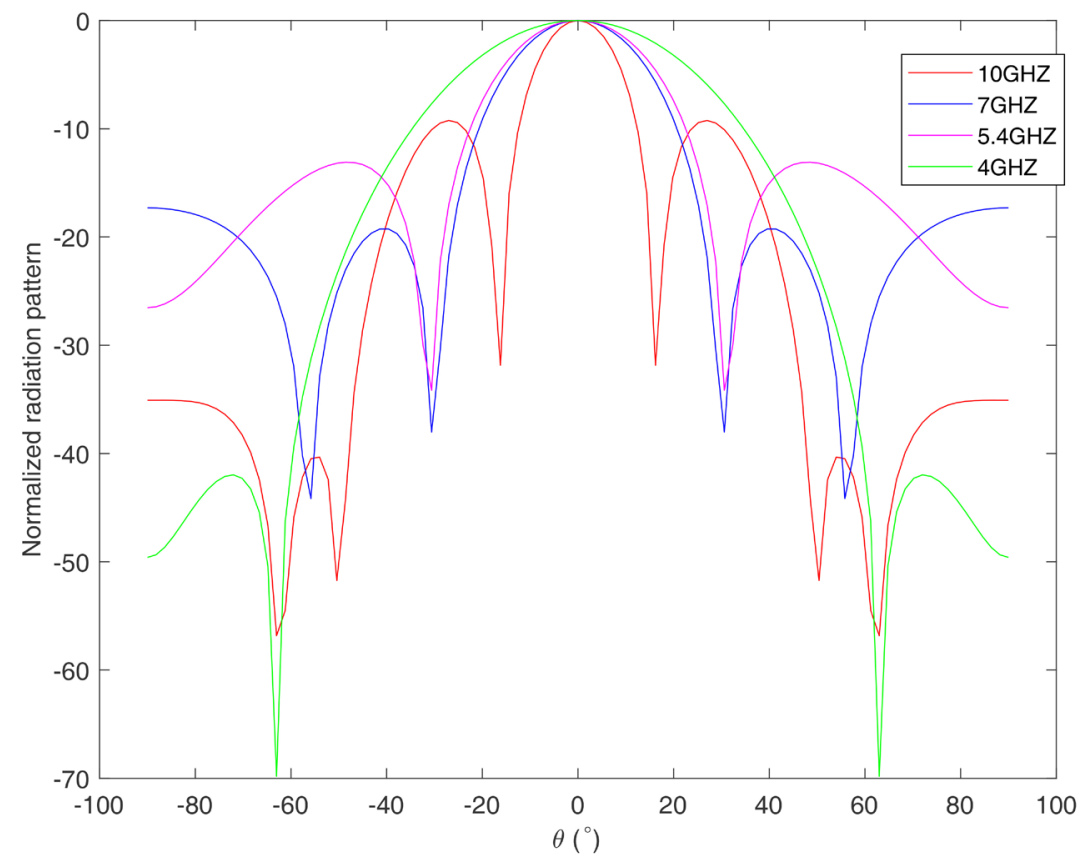

Figure 6. The normalized radiation pattern in value $(\mathrm{dB})$ against several frequencies $F$ using: $h=1.25 \mathrm{~mm}, d_{x}=54 \mathrm{~mm}, \quad d_{y}=54 \mathrm{~mm}, \quad w=1 \mathrm{~mm}, \quad \epsilon=1, \quad \epsilon_{r}=1$, $\delta=0.75 \mathrm{~mm}, \lambda=54 \mathrm{~mm}, \quad \alpha=0 \mathrm{rad} \cdot \mathrm{m}^{-1}, \quad \beta=0 \mathrm{rad} \cdot \mathrm{m}^{-1}$. 


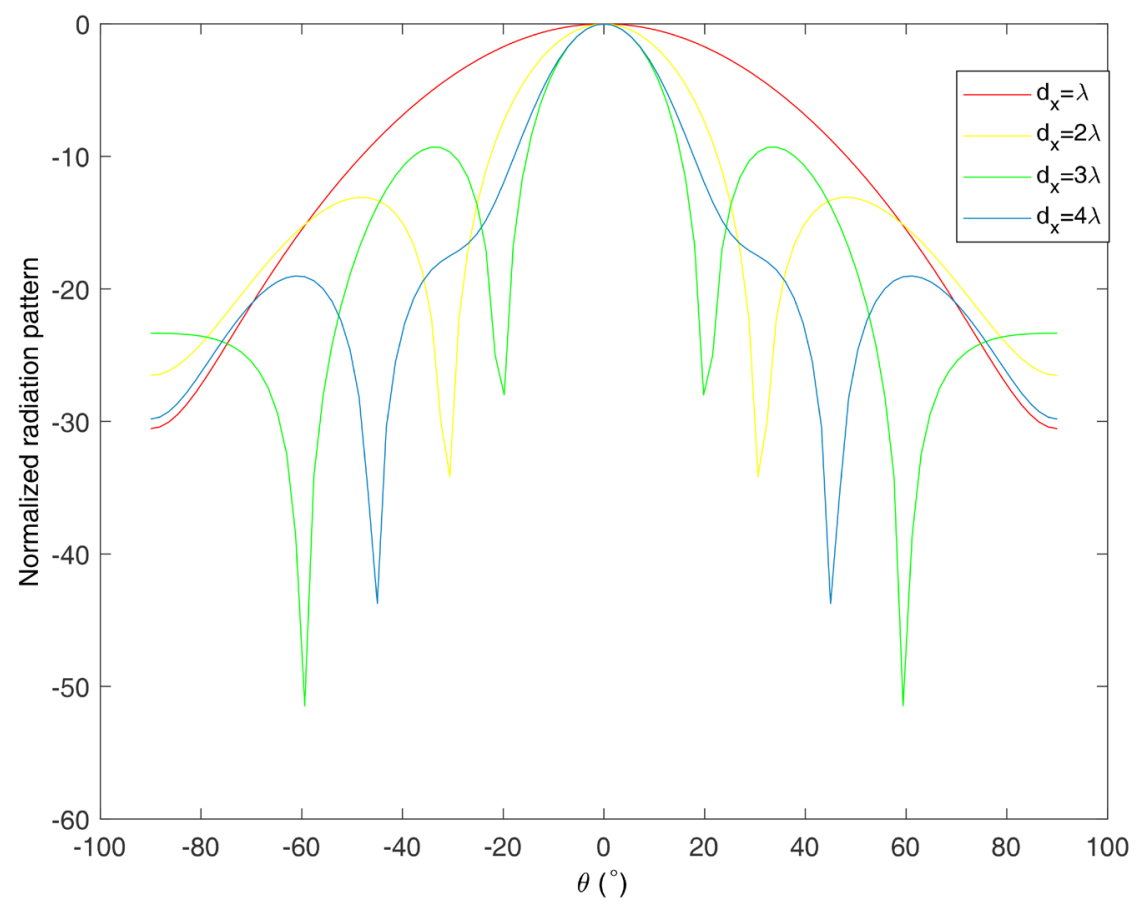

Figure 7. The normalized radiation pattern value in $(\mathrm{dB})$ against several periods $d_{x}$ of global structure using: $F=5.4 \mathrm{Ghz}, h=1.25 \mathrm{~mm}, d_{y}=54 \mathrm{~mm}, w=1 \mathrm{~mm}, \quad \epsilon=1$, $\epsilon_{r}=1, \delta=0.75 \mathrm{~mm}, \lambda=54 \mathrm{~mm}, \alpha=0 \mathrm{rad} \cdot \mathrm{m}^{-1}, \quad \beta=0 \mathrm{rad} \cdot \mathrm{m}^{-1}$.

\section{3-D radiation of Smart Periodic Antenna}

\subsection{Coupling Effect}

In this section, we study the influence of the coupling and the steering direction on the electromagnetic parameters of our periodic structure.

Figure 8 shows a 3-D beam radiation of the coupled structure in the steered direction $\theta_{s}=0$ for different coupling states $d_{x}=\lambda, d_{x}=2 \lambda, d_{x}=3 \lambda, d_{x}=4 \lambda$. Electromagnetic performance parameters of this coupled structure in different coupling values are shown in Table 2.

\subsection{Steering Directions Effect}

Figure 9 shows a 3-D beam radiation of the coupled structure in the high coupling value $\left(d_{x}=2 \lambda\right)$ for different steering directions:

$$
\theta_{s}=0, \theta_{s}=\Pi / 6, \theta_{s}=\Pi / 4, \theta_{s}=\Pi / 3 .
$$

Electromagnetic performance parameters of this coupled structure in different steered directions $\theta_{s}$ are shown in Table 3 for high and low coupling values.

In the previous section, we evaluated periodic antennas with uniform spatial periodicity and equitable amplitude distribution. The study of coupling and angular scanning leads us to design an intelligent antenna with a sweeping beam. The purpose of this design is to lead the radiation to the desired direction of space without affecting the radiation characteristics. The effect of the secondary lobes in the antenna radiation pattern is virtually undesirable because it affects 


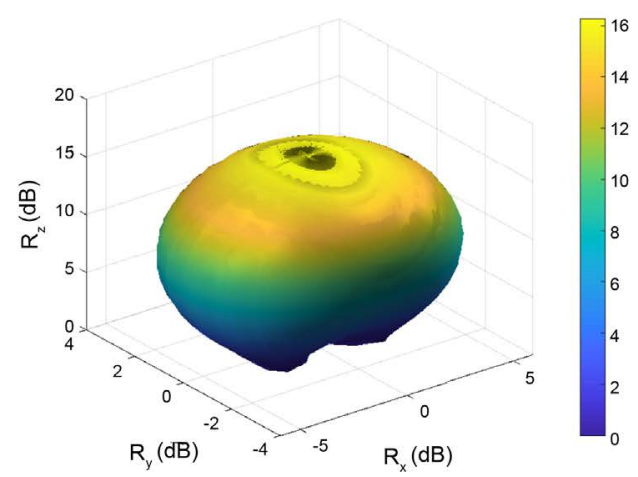

(a)

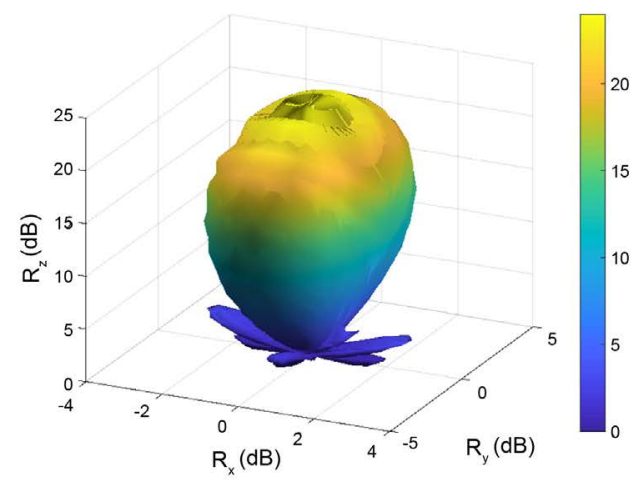

(c)

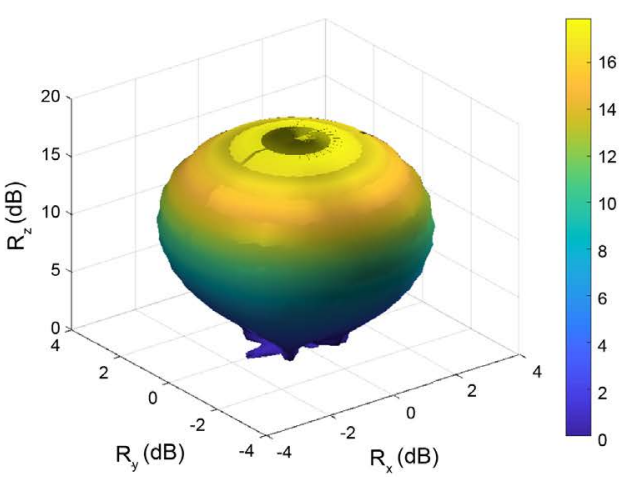

(b)

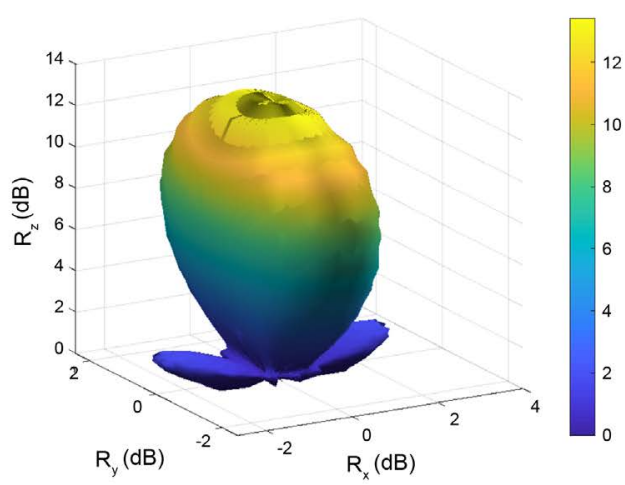

(d)

Figure 8. The 3-D radiation beam pattern of proposed antenna array at different coupling states for steered angle $\theta_{s}=0$ using: $F=10.4 \mathrm{Ghz}, h=1.25 \mathrm{~mm}, d_{y}=54 \mathrm{~mm}, w=1 \mathrm{~mm}, \epsilon=1, \epsilon_{r}=1, \delta=0.75 \mathrm{~mm}$, $\lambda=28 \mathrm{~mm} ;$ (a) $d_{x}=\lambda$; (b) $d_{x}=2 \lambda$; (c) $d_{x}=3 \lambda$; (d) $d_{x}=4 \lambda$.

Table 2. The Performance Parameters for Different Coupling Values $d_{x}$ of the 1-D periodic structure.

\begin{tabular}{cc}
\hline Periodicity $d_{x}$ & Directivity $(\mathrm{dB})$ \\
\hline$\lambda$ & 29.48 \\
$2 \lambda$ & 30.38 \\
$3 \lambda$ & 35.11 \\
$4 \lambda$ & 39.99 \\
\hline
\end{tabular}

Table 3. The performance parameters for different steering directions $\theta_{s}$ in in low and high coupling values of $1-\mathrm{D}$ periodic structure.

\begin{tabular}{ccc}
\hline Steering angle $\theta_{s}$ & $\begin{array}{c}\text { Directivity (dB) low coupling } \\
\left(d_{x}=4 \lambda\right)\end{array}$ & $\begin{array}{c}\text { Directivity (dB) high coupling } \\
\left(d_{x}=\lambda\right)\end{array}$ \\
\hline 0 & 39.99 & 29.48 \\
$\Pi / 6$ & 28.10 & 20.41 \\
$\Pi / 4$ & 24.66 & 19.51 \\
$\Pi / 3$ & 21.44 & 19.37 \\
\hline
\end{tabular}




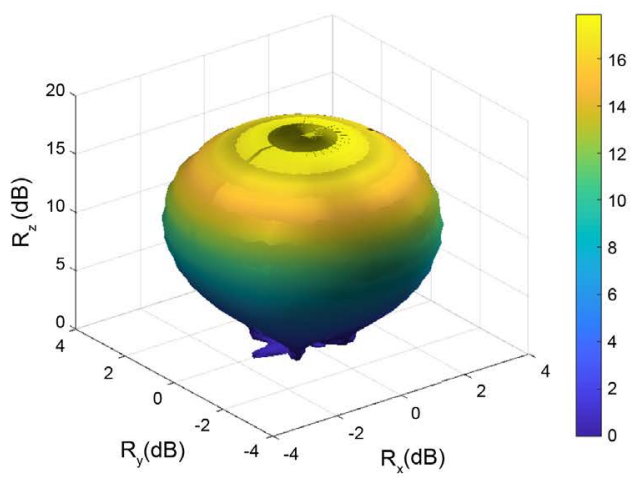

(a)

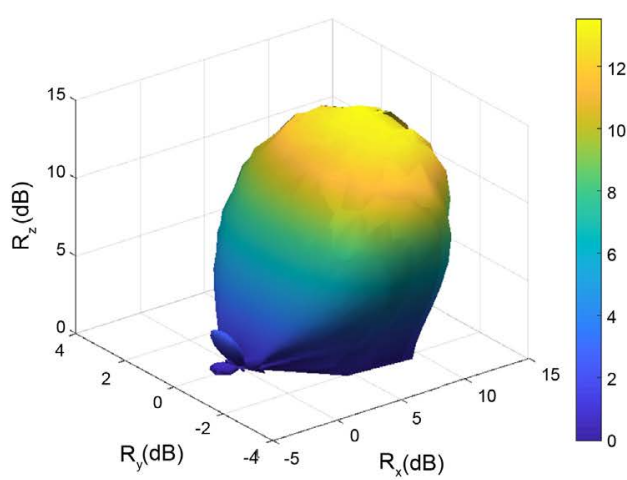

(c)

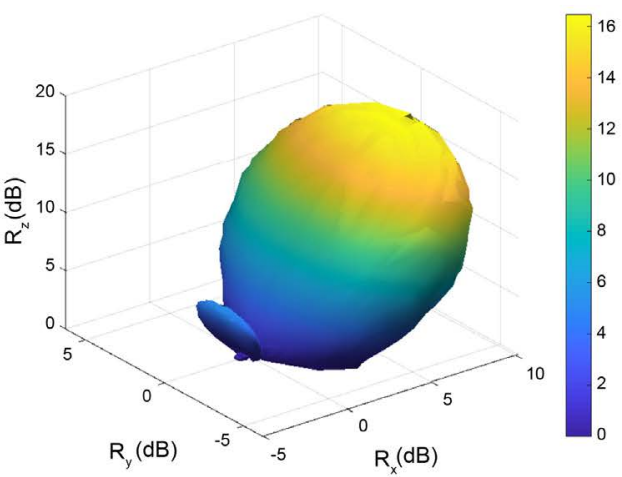

(b)

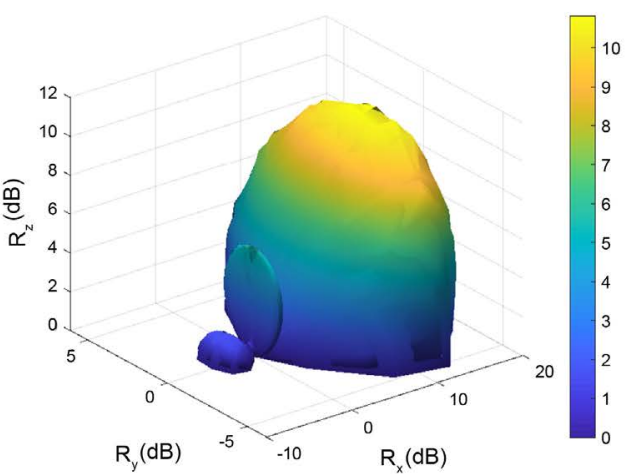

(d)

Figure 9. The 3-D radiation beam pattern of proposed antenna array at different steering directions $\theta_{s}$ in high coupling values using: $F=5.4 \mathrm{Ghz}, \lambda=28 \mathrm{~mm}, h=1.25 \mathrm{~mm}, d_{x}=2 \lambda, d_{y}=54 \mathrm{~mm}, w=1 \mathrm{~mm}$, $\epsilon=1, \epsilon_{r}=1, \delta=0.75 \mathrm{~mm}$; (a) $\theta_{s}=0$, (b) $\theta_{s}=\Pi / 6$, (c) $\theta_{s}=\Pi / 4$, (d) $\theta_{s}=\Pi / 3$.

the main lobe gain by energy dispersal and also disturbs the second radiant element. Therefore, a condition regarding the spacing of sources must be imposed.

Figure 10 represents respectively the variation of the directivity and the side lobe level for several distances $d$ and for several steering directions $\theta_{s}$. For the working frequency $F=10 \mathrm{GHz}$, the spacing $d$ between the sources must exceed $2 \lambda=56 \mathrm{~mm}$ to ensure a $-18.3 \mathrm{~dB}$ of side lobe level. Also, for the same parameters, to obtain a directivity greater than $23.42 \mathrm{~dB}$ the steering angle must not exceed pi/6. By exploiting Figure 10, we can concept a smart periodic antenna with minimal coupling and acceptable directivity. finally, we can deduce that high directivity values can be achieved with low sweeping angles $\theta_{s}$, also low coupling values can be achieved with high spacing values $d$.

\section{Conclusion}

In this contribution, we have presented a theoretical analysis of 1-D and 2-D periodic antennas. A novel modal approach combined with MoM-GEC was used. The behavior of pattern radiation of the coupled reference cell has been illustrated corresponding to different frequency ranges, period value and Floquet 


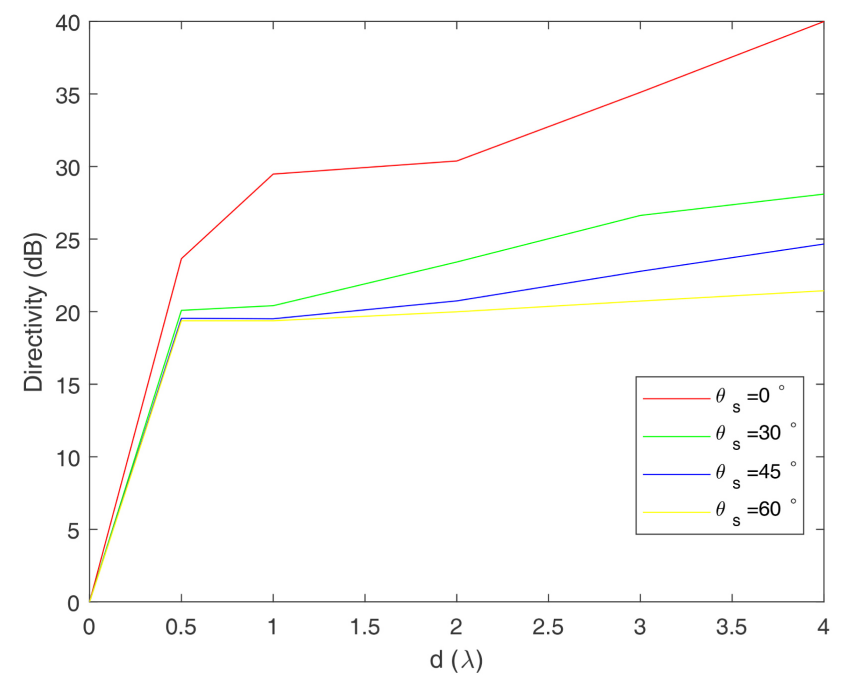

(a)

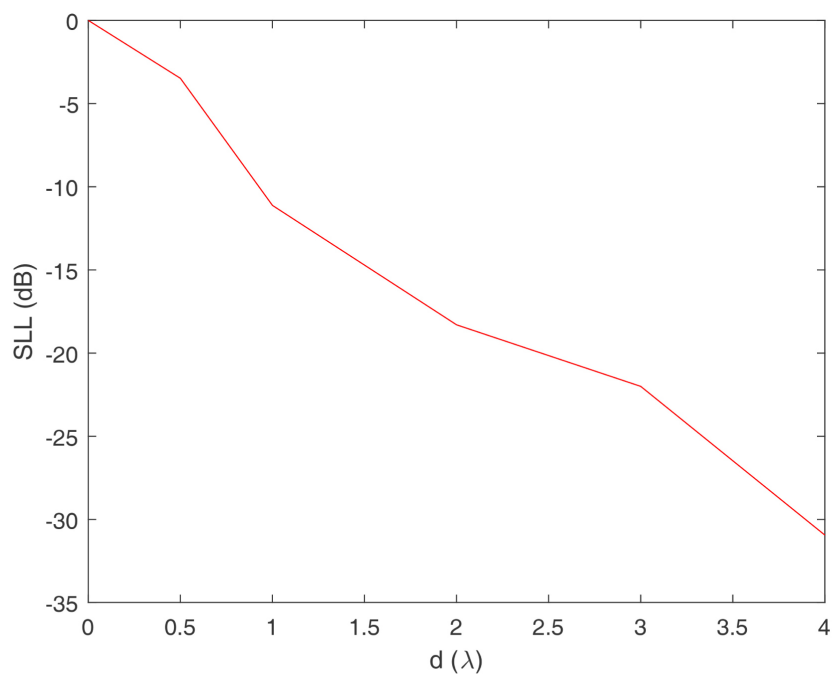

(b)

Figure 10. Directivity and side lobe level evolution of proposed antenna array at different steering directions $\theta_{s}: F=10.4 \mathrm{Ghz}$, $h=1.25 \mathrm{~mm}, d_{y}=54 \mathrm{~mm}, w=1 \mathrm{~mm}, \epsilon=1, \epsilon_{r}=1, \delta=0.75 \mathrm{~mm}, \lambda=28 \mathrm{~mm}$; (a) directivity in terms of $\lambda$, (b) SLL in terms of $\lambda$.

modes. To properly control the directivity of periodic antennas and minimize coupling between cells, it is necessary to choose the best period $d_{x}$. This can be also useful for the analysis of 3-D beam radiation for different steering directions and coupling values. Then, we can concept a smart periodic antenna with minimal coupling and acceptable directivity for different sweeping angles. For the special case when high coupling is presented, we can easily show that directivity is reduced to $19.37 \mathrm{~dB}$ when $\theta_{s}=\pi / 3$, while the side lobe level is increased to $-11.12 \mathrm{~dB}$. The essential advantage of this new modal analysis is concepting coupled periodic antenna with sweeping radiation beam. The numerical results demonstrate the feasibility of the proposed approach in the field of estimation side lobe level and directivity of almost periodic antenna. In conclusion, we would like to highlight that Floquet model analysis and MoM-GEC method are needed to study coupled smart antenna that opens various areas of research.

\section{Conflicts of Interest}

The authors declare no conflicts of interest regarding the publication of this paper.

\section{References}

[1] Baudrand, H., Titaouine, M., Raveu, N. and Fontgland, G. (2009) Electromagnetic Modeling of Planar almost Periodic Structures. SBMOII IEEE MTT-S International Microwave and Optoelectronics Conference, Belem, 3-6 November 2009, 427-431. https://doi.org/10.1109/IMOC.2009.5427552

[2] Mikkioui, Z. and Baudrand, H. (2008) Bi-Dimensional Bi-Periodic Centred Field Microstrip Leakly Wave Antenna Analysis by a Source Modal Decomposition in Sperctal Domain. IET Microwaves Antennas and Propagation, 3, 1141-1149.

[3] Mekkioui, Z. and Baudrand, H. (2008) Full-Wave Analysis of Uniform Microstrip 
Leaky-Wave Antenna with Arbitrary Metallic Strips. Electromagnetic, 28, 296-314. https://doi.org/10.1080/02726340802040161

[4] Xi, Y.P., Fang, D.G., Sun, Y.X. and Chow, Y.L. (2005) Mutual Coupling in a Linear Dipole Array of Finit Size. IEE Proceedings-Microwaves Antennas and Propagation, 152, 324-330.

[5] Massaro, A., Cingolani, A., Passasea, A. and Vittoriol, M. (2009) Floquet's Unit Cell Deseign for Periodic Structures Atoptical Frequencies. International Journal of Microwave Science and Technology, 2009, Article ID 160321. https://doi.org/10.1155/2009/160321

[6] Bhattacharyya, K.A. (2012) Floquet Modal Based Analysis of Finite and Infinite Phased Array Antennas. Macquarie University and IEEE Joint Lecture.

[7] Arun Bhattacharyya, K. (2006) Phased Array Antennas. John Wiley and Sos, Inc. Publication, Hoboken.

[8] Makarov, S., Puzella, A. and Iyer, V. (2008) Scan Impedance for an Infinite Dipole Array: Accurate Theoretical Model Compared to Numerical Software. IEEE Antennas and Propagation Magazine, 50, 132-149. https://doi.org/10.1109/MAP.2008.4768944

[9] Sze, K. and Shafai, L. (1999) Reflection Properties of Infinite Periodic Arrays of Rectangular Conducting Patches. Canadian Journal of Electrical and Computer Engineering, 24, 35-41.

[10] Watanable, K. and Yasumoto, K. (2007) Tow-Dimensional Electromagnetic Scattering of Non-Plane Incident Waves by Periodic Structures. Progress in Electromagnetics Research, 74, 241-271. https://doi.org/10.2528/PIER07050902

[11] Skrivervik, K. and Mosig, L. (1992) Finite Phased Array of Microstrip Patch Antennas: The Infinite Array Approach. IEEE Transactions on Antennas and Propagation, 40, 579-582.

[12] Reyanaa, A., Panduroa, D., Covarrubias and Mendeza, A. (2012) Design of Steerable Concentric Rings Array for Low Side Lobe Level. Scientia Iranica, 19, 727-732. https://doi.org/10.1016/j.scient.2011.08.028

[13] Dauguet, S., Gillard, R., Citerne, J. and Piton, G. (1997) Extension of the Compression Approach to Include the Treatement of Radiation Pattern in the Electromagnetic Analysis of Active Plannar Antennas. Antennas and Propagation Society International Symposium, 22-25.

[14] Bilel, H., Aguili, T. and Baugrand, H. (2015) Floquet Modal Analysis to Modelize and Study 2 D Planar almost Periodic Structures in Finite and Infinite Extend with Coupled Motifs. PIERS B.

[15] Bilel, H., Aguili, T., Ravue, N. and Baugrand, H. (2014) Calculation of Mutual Coupling Parameter and Their Effects in 1D almost Periodic Structures. PIERS B.

[16] Bilel, H., Limam, S. and Aguili, T. (2016) Uniform and Concentric Circular Antenna Arrays Synthesis for Smart Antenna Systems Using Artificial Neural Network Algorithm. Progress in Electromagnetics Research B, 67, 91-105. https://doi.org/10.2528/PIERB16031508

[17] Bilel, H., Aguili, T. and Audrand, H. (2012) Uni-Dimensional Planar Almost Periodic Structures Analysis to Decompose Central Arbitrary Located Source in Spectral Domain. International Symposium on Antenna Technology and Applied Electromagnetics, Toulouse, 25-28 June 2012, 1-5.

[18] Rodrguez-Berral, R., Mesa, F., Baccarelli, P. and Burghignoli, P. (2009) Excitation of 
a Periodic Microstrip Line by an Aperiodic Delta-Gap Source. IEEE Transactions on Antennas and Propagation, 8, 641-644.

https://doi.org/10.1109/LAWP.2009.2023252

[19] Berry, D., Malech, R. and Kennedy, W. (2012) The Reflectarray Antenna. IEEE Transaction on Antennas and Propagation, 11, 645-651.

https://doi.org/10.1109/TAP.1963.1138112

[20] Riabi, M., Ahmadpanah, M., Benzina, H., Baudrand, H. and Fouad Hanna, V. (1995) Performance of the LSBRM Using Efficient Weighting Functions for Planar Structures. IEEE Transaction on Antennas and Propagation, 142, 364-368. https://doi.org/10.1049/ip-map:19952004

[21] Mili, S. and Aguili, T. (2011) Study of Fractal-Shaped Structures with Pin Diodes Using the Multi-Scale Method Combined to the Generalized Equivalen Circuit Modeling. Progress in Electromagnetics Research B, 27, 213-233. https://doi.org/10.2528/PIERB10110105

[22] Montgomery, J. (1978) Scattering by an Infinite Periodic Array of Microstrip Elements? IEEE Transaction on Antennas and Propagation, 26, 850-854. https://doi.org/10.1109/TAP.1978.1141956

[23] Arrebola, M., Alvarez, Y., Encinar, J. and Las-Heras, F. (2009) Accurate Analysis of Printed Reflectarrays Considering the near Field of the Primary Feed. IET Microwaves, Antennas and Propagation, 3, 187-194.

https://doi.org/10.1049/iet-map:20070325

[24] Hannan, P. and Balfour, M. (1965) Simulation of a Phased Array Antenna in Waveguide. IEEE Transaction on Antennas and Propagation, 13, 342-353. https://doi.org/10.1109/TAP.1965.1138428

[25] Milon, M., Cadoret, D., Gillard, R. and Legay, H. (2007) Surrounded-Element Approach for the Simulation of Reflectarray Radiating Cells. IET Microwaves Antennas and Propagation, 1, 289-293. https://doi.org/10.1049/iet-map:20050291

[26] Aguili, T. (2000) Thesis Modélisation des composants SHF planaires par la mthodecircuits equivalents. National Engineering Scool of Tunis ENIT.

[27] Aubert, H. and Baudrant, H. (2003) Lelectromagnetisme par les Schemas Equivalents. Cepadus.

[28] Raveu, N. and Pigaglio, O. (2012) Rsolution de problemes hautes frquences par les schmas quivalents. ditions Cpadues.

[29] Clement, Y. (2012) Thesis Modlisation lectromagntique de cellules actives environnes-Application a l'analyse et la synthese d'une antenne reflectarray a balayage lecronique. INSA de Rennes.

[30] Jihed, B.S. (2013) Rductibilit et thorie de Floquet pour systemes diffrenciels non linaires. Universit Panthon-Sorbone, Paris.

[31] Nosal, S. (2009) Modlisation lectromagntique de structures priodiques et matriaux artificiels Application a la conception d'un radome passe bande. cole centrale Paris.

[32] Reynaa, A., Panduroa, A. and Covarrubias, H. (2012) Design of Steerable Concentric Rings Array for Low Side Lobe Level. Scientia Iranica, 19, 727-732. https://doi.org/10.1016/j.scient.2011.08.028

[33] Ben Latifa, N., Bilel, H. and Aguili, T. (2017) Floque Modal Analysis to Study Radiation Pattern for Coupled Almost Periodic Antenna Array. 14th International Conference on Computer Systems and Applications, Hammamet, 30 October-3 November 2017, 109-113. 
[34] Ben Latifa, N., Bilel, H. and Aguili, T. (2017) Floque Modal Analysis to Study Periodic and Aperiodic Planar Antenne Arrays with Mututal Coupling.

[35] Ben Latifa, N., Bilel, H. and Aguili, T. (2018) Electronically Steerable Radiation Pattern of Coupled Periodic Antenna Used Floquet Analysis.

[36] Ben Latifa, N., Bilel, H. and Aguili, T. (2018) Three-Dimensional Radiation of Coupled Smart Periodic Antennas Using Floquet Modal Analysis. 